\title{
Existence of Solutions of Three-Dimensional Fractional Differential Systems
}

\author{
Vadivel Sadhasivam, Jayapal Kavitha, Muthusamy Deepa \\ Post Graduate and Research Department of Mathematics, Thiruvalluvar Government Arts College (Affli. to Periyar University), \\ Rasipuram, India \\ Email: ovsadha@gmail.com, kaviakshita@gmail.com, mdeepa.maths@gmail.com
}

How to cite this paper: Sadhasivam, V., Kavitha, J. and Deepa, M. (2017) Existence of Solutions of Three-Dimensional Fractional Differential Systems. Applied Mathematics, 8, 193-208. https://doi.org/10.4236/am.2017.82016

Received: January 6, 2017

Accepted: February 24, 2017

Published: February 27, 2017

Copyright (c) 2017 by authors and Scientific Research Publishing Inc. This work is licensed under the Creative Commons Attribution International License (CC BY 4.0).

http://creativecommons.org/licenses/by/4.0/

\section{cC) () Open Access}

$$
\begin{aligned}
& \text { Abstract } \\
& \text { In this article, we consider the three-dimensional fractiona } \\
& \text { of the form } \\
& \qquad \begin{array}{l}
D_{0^{+}}^{\alpha} u(t)=f_{1}\left(t, v(t), v^{\prime}(t)\right), t \in(0,1), \\
D_{0^{+}}^{\beta} v(t)=f_{2}\left(t, w(t), w^{\prime}(t)\right), t \in(0,1), \\
D_{0^{+}}^{\gamma} w(t)=f_{3}\left(t, u(t), u^{\prime}(t)\right), t \in(0,1),
\end{array}
\end{aligned}
$$

In this article, we consider the three-dimensional fractional differential system

together with the Neumann boundary conditions,

$$
u^{\prime}(0)=u^{\prime}(1)=0, v^{\prime}(0)=v^{\prime}(1)=0, w^{\prime}(0)=w^{\prime}(1)=0,
$$

where $D_{0^{+}}^{\alpha}, D_{0^{+}}^{\beta}, D_{0^{+}}^{\gamma}$ are the standard Caputo fractional derivatives, $1<\alpha, \beta, \gamma \leq 2$. A new result on the existence of solutions for a class of fractional differential system is obtained by using Mawhin's coincidence degree theory. Suitable examples are given to illustrate the main results.

\section{Keywords}

Fractional Differential Equations, Boundary Value Problem, Coincidence Degree Theory

\section{Introduction}

Fractional calculus is a very effective tool in the modeling of many phenomena like control of dynamical systems, porous media, electro chemistry, viscoelasticity, electromagnetic and so on. The fractional theory and its applications are mentioned by many papers and monographs, we refer [1]-[9]. For nonlinear fractional boundary value problem, many fixed point theorems were applied to investigate the existence of solutions as in references [10] [11] [12] [13]. On the other hand, there is another effective approach, Mawhin's coincidence theory, which proves to be very useful for determining the existence of solutions for 
fractional order differential equations. In recent years, boundary value problems for fractional differential equations at resonance have been studied in many papers (see [14]-[21]). The main motivation for investigating the fractional boundary value problem arises from fractional advection-dispersion equation.

$\mathrm{Hu}$ et al. [22] investigated the two-point boundary value problem for fractional differential equations of the following form

$$
\begin{aligned}
& D_{0^{+}}^{\alpha} x(t)=f\left(t, x(t), x^{\prime}(t)\right), t \in[0,1], \\
& x(0)=0, x^{\prime}(0)=x^{\prime}(1),
\end{aligned}
$$

where $D_{0^{+}}^{\alpha}$ is the Caputo fractional differential operator, $1<\alpha \leq 2$, and $f:[0,1] \times R^{2} \rightarrow R$ is continuous.

In [23], Hu et al. extended the above boundary value problem to the existence of solutions for the following coupled system of fractional differential equations of the form

$$
\begin{aligned}
& D_{0^{+}}^{\alpha} u(t)=f\left(t, v(t), v^{\prime}(t)\right), t \in(0,1), \\
& D_{0^{+}}^{\beta} v(t)=g\left(t, u(t), u^{\prime}(t)\right), t \in(0,1), \\
& u(0)=v(0)=0, u^{\prime}(0)=u^{\prime}(1), v^{\prime}(0)=v^{\prime}(1) .
\end{aligned}
$$

where $D_{0^{+}}^{\alpha}, D_{0^{+}}^{\beta}$ are the Caputo fractional derivatives, $1<\alpha \leq 2$, $1<\beta \leq 2$, and $f, g:[0,1] \times R^{2} \rightarrow R$ is continuous.

It seems that there has been no work done on the boundary value problem of system involving three nonlinear fractional differential equations. Motivated by the above observation, we investigate the following three-dimensional fractional differential system of the form

$$
\begin{aligned}
& D_{0^{+}}^{\alpha} u(t)=f_{1}\left(t, v(t), v^{\prime}(t)\right), t \in(0,1), \\
& D_{0^{+}}^{\beta} v(t)=f_{2}\left(t, w(t), w^{\prime}(t)\right), t \in(0,1), \\
& D_{0^{+}}^{\gamma} w(t)=f_{3}\left(t, u(t), u^{\prime}(t)\right), \quad t \in(0,1),
\end{aligned}
$$

together with the Neumann boundary conditions,

$$
u^{\prime}(0)=u^{\prime}(1)=0, v^{\prime}(0)=v^{\prime}(1)=0, w^{\prime}(0)=w^{\prime}(1)=0,
$$

where $D_{0^{+}}^{\alpha}, D_{0^{+}}^{\beta}, D_{0^{+}}^{\gamma}$ are the standard Caputo fractional derivatives, $1<\alpha, \beta, \gamma$ $\leq 2$, and $f_{1}, f_{2}, f_{3}:[0,1] \times R \times R \rightarrow R$ is continuous.

The main goal of this paper is to establish some new criteria for the existence of solutions of (1). The method is based on Mawhin's coincidence degree theory. The results in this paper are generalized of the existing ones.

\section{Preliminaries}

In this section, we give the definitions of fractional derivatives and integrals and some notations which are useful throughout this paper. There are several kinds of definitions of fractional derivatives and integrals. In this paper, we use the Riemann-Liouville left sided definition on the half-axis $R_{+}$and the Caputo fractional derivative.

Let $X$ and $Y$ be real Banach spaces and let $L: \operatorname{dom} L \subset X \rightarrow Y$ be a Fred- 
holm operator with index zero if $\operatorname{dim} \operatorname{Ker} L=\operatorname{codim} \operatorname{Im} L<\infty$ and $\operatorname{Im} L$ is closed in $Y$ and there exist continuous projectors $P: X \rightarrow X, Q: Y \rightarrow Y$ such that $\operatorname{Im} P=\operatorname{Ker} L$, Ker $Q=\operatorname{Im} L, X=\operatorname{Ker} L \oplus \operatorname{Ker} P, Y=\operatorname{Im} L \oplus \operatorname{Im} Q$.

It follows that

$$
\left.L\right|_{\operatorname{dom} L \cap \operatorname{Ker} P}: \operatorname{dom} L \cap \operatorname{Ker} P \rightarrow \operatorname{Im} L
$$

is invertible. Here $K_{P}$ denotes the inverse of $\left.L\right|_{\operatorname{dom} L \cap \operatorname{Ker} P}$.

If $\Omega$ is an open bounded subset of $X$, and $\operatorname{dom} L \cap \bar{\Omega} \neq \phi$, then the map $N: X \rightarrow Y$ will be called L-compact on $\bar{\Omega}$, if $Q N(\bar{\Omega})$ is bounded and $K_{P}(I-Q) N: \bar{\Omega} \rightarrow X$ is compact, where $I$ is the identity operator.

Lemma 1. [14] Let $L: \operatorname{dom} L \subset X \rightarrow Y$ be a Fredholm operator with index zero and $N: X \rightarrow Y$ be L-compact on $\bar{\Omega}$. Assume that the following conditions are satisfied.

1. $L x \neq \lambda N x$ for every $(x, \lambda) \in[(\operatorname{dom} L \backslash \operatorname{Ker} L) \cap \partial \Omega] \times(0,1)$;

2. $N x \notin \operatorname{Im} L$ for every $x \in \operatorname{Ker} L \cap \partial \Omega$;

3. $\operatorname{deg}\left(\left.Q N\right|_{\operatorname{Ker} L}, \operatorname{Ker} L \cap \Omega, 0\right) \neq 0$, where $Q: Y \rightarrow Y$ is a projection such that $\operatorname{Im} L=\operatorname{Ker} Q$.

Then the operator equation $L x=N x$ has at least one solution in $\operatorname{dom} L \cap \bar{\Omega}$.

Definition 1. [6] The Riemann-Liouville fractional integral of order $\alpha>0$ of a function $y: R_{+} \rightarrow R$ on the half-axis $R_{+}$is given by

$$
\left(I_{0^{+}}^{\alpha} y\right)(t):=\frac{1}{\Gamma(\alpha)} \int_{0}^{t}(t-v)^{\alpha-1} y(v) \mathrm{d} v \text { for } t>0
$$

provided the right hand side is pointwise defined on $R_{+}$.

Definition 2. [6] Assume that $x(t)$ is $(n-1)$-times absolutely continuous function, the Caputo fractional derivative of order $\alpha>0$ of $x$ is given by

$$
\left(D_{0^{+}}^{\alpha} x\right)(t):=I_{0^{+}}^{n-\alpha} \frac{\mathrm{d}^{n} x(t)}{\mathrm{d} t^{n}}=\frac{1}{\Gamma(n-\alpha)} \int_{0}^{t}(t-v)^{n-\alpha-1} x^{(n)}(v) \mathrm{d} v \text { for } t>0
$$

where $n$ is the smallest integer greater than or equal to $\alpha$, provided that the right side integral is pointwise defined on $(0,+\infty)$.

Lemma 2. [6] Let $\alpha>0$ and $n=-[-\alpha]$. If $x^{(n-1)} \in A C[0,1]$, then

$$
I_{0^{+}}^{\alpha} D_{0^{+}}^{\alpha} x(t):=x(t)+c_{0}+c_{1} t+c_{2} t^{2}+\cdots+c_{n-1} t^{n-1}
$$

where $c_{i}=-\frac{x^{(i)}(0)}{i !} \in R, i=0,1,2, \cdots, n-1$, here $n$ is the smallest integer greater than or equal to $\alpha$.

In this paper, let us take $X=C^{1}[0,1]$ with the norm $\|x\|_{X}=\max \left\{\|x\|_{\infty},\left\|x^{\prime}\right\|_{\infty}\right\}$ and $Y=C[0,1]$ with the norm $\|y\|_{Y}=\|y\|_{\infty}$, where $\|x\|_{\infty}=\max _{t \in[0,1]}|X(t)|$. Then we denote $\bar{X}=X \times X \times X$ with the norm $\|(u, v, w)\|_{\bar{X}}=\max \left\{\|u\|_{X},\|v\|_{X},\|w\|_{X}\right\}$ and $\bar{Y}=Y \times Y \times Y$ with the norm $\|(x, y, z)\|_{\bar{Y}}=\max \left\{\|x\|_{Y},\|y\|_{Y},\|z\|_{Y}\right\}$. Clearly, both $\bar{X}$ and $\bar{Y}$ are Banach spaces.

Define the operators $L_{i}: \operatorname{dom} L \subset X \rightarrow Y,(i=1,2,3)$ by 


$$
L_{1} u=D_{0^{+}}^{\alpha} u, L_{2} v=D_{0^{+}}^{\beta} v \text { and } L_{3} w=D_{0^{+}}^{\gamma} w \text {, }
$$

where

$$
\begin{aligned}
& \operatorname{dom} L_{1}=\left\{u \in X \mid D_{0^{+}}^{\alpha} u(t) \in Y, u^{\prime}(0)=u^{\prime}(1)=0\right\}, \\
& \operatorname{dom} L_{2}=\left\{v \in X \mid D_{0^{+}}^{\beta} v(t) \in Y, v^{\prime}(0)=v^{\prime}(1)=0\right\},
\end{aligned}
$$

and

$$
\operatorname{dom} L_{3}=\left\{w \in X \mid D_{0^{+}}^{\gamma} w(t) \in Y, w^{\prime}(0)=w^{\prime}(1)=0\right\} .
$$

Define the operator $L: \operatorname{dom} L \subset \bar{X} \rightarrow \bar{Y}$ by

$$
L(u, v, w)=\left(L_{1} u, L_{2} v, L_{3} w\right),
$$

where $\operatorname{dom} L=\left\{(u, v, w) \in \bar{X} \mid u \in \operatorname{dom} L_{1}, v \in \operatorname{dom} L_{2}, w \in \operatorname{dom} L_{3}\right\}$.

Let the Nemytski operator $N: \bar{X} \rightarrow \bar{Y}$ be defined as

$$
N(u, v, w)=\left(N_{1} v, N_{2} w, N_{3} u\right),
$$

where $N_{1}: X \rightarrow Y$ is defined by

$$
N_{1} v(t)=f_{1}\left(t, v(t), v^{\prime}(t)\right),
$$

$N_{2}: X \rightarrow Y$ is defined by

$$
N_{2} w(t)=f_{2}\left(t, w(t), w^{\prime}(t)\right),
$$

and $N_{3}: X \rightarrow Y$ is defined by

$$
N_{3} u(t)=f_{3}\left(t, u(t), u^{\prime}(t)\right) .
$$

Then Neumann boundary value problem (1) is equivalent to the operator equation

$$
L(u, v, w)=N(u, v, w), \quad(u, v, w) \in \operatorname{dom} L .
$$

\section{Main Results}

In this section, we begin with the following theorem on existence of solutions for Neumann boundary value problem (1).

Theorem 1. Let $f_{1}, f_{2}, f_{3}:[0,1] \times R \times R \rightarrow R$ be continuous. Assume that

(H1) there exist nonnegative functions $a_{i}, b_{i}, c_{i} \in C[0,1],(i=1,2,3)$ with

$$
\frac{\Gamma(\alpha) \Gamma(\beta) \Gamma(\gamma)-\left(B_{1}+C_{1}\right)\left(B_{2}+C_{2}\right)\left(B_{3}+C_{3}\right)}{\Gamma(\alpha) \Gamma(\beta) \Gamma(\gamma)}>0
$$

such that for all $(u, v) \in R^{2}, t \in[0,1]$,

$$
\left|f_{i}(t, u, v)\right| \leq a_{i}(t)+b_{i}(t)|u|+c_{i}(t)|v| \text { for } i=1,2,3,
$$

where $A_{i}=\left\|a_{i}\right\|_{\infty}, B_{i}=\left\|b_{i}\right\|_{\infty}, C_{i}=\left\|c_{i}\right\|_{\infty}, \quad(i=1,2,3)$;

(H2) there exists a constant $M>0$ such that for all $t \in[0,1],|u|>M, v \in R$ either

$$
u f_{1}(t, u, v)>0, u f_{2}(t, u, v)>0, u f_{3}(t, u, v)>0
$$

or 


$$
u f_{1}(t, u, v)<0, u f_{2}(t, u, v)<0, u f_{3}(t, u, v)<0 ;
$$

(H3) there exists a constant $M^{*}>0$ such that for every $m_{1}, m_{2}, m_{3} \in R$ satisfying $\min \left\{m_{1}, m_{2}, m_{3}\right\}>M^{*}$ either

$$
m_{1} N_{1}\left(m_{2}\right)>0, m_{2} N_{2}\left(m_{3}\right)>0, m_{3} N_{3}\left(m_{1}\right)>0
$$

or

$$
m_{1} N_{1}\left(m_{2}\right)<0, m_{2} N_{2}\left(m_{3}\right)<0, m_{3} N_{3}\left(m_{1}\right)<0 .
$$

Then Neumann boundary value problem (1) has at least one solution.

Lemma 3. Let $L$ be defined by (2). Then

$$
\text { Ker } \begin{aligned}
L & =\left(\operatorname{Ker} L_{1}, \operatorname{Ker} L_{2}, \operatorname{Ker} L_{3}\right) \\
& =\{(u, v, w) \in \bar{X} \mid(u, v, w)=(u(0), v(0), w(0))\},
\end{aligned}
$$

and

$$
\begin{aligned}
\operatorname{Im} L & =\left(\operatorname{Im} L_{1}, \operatorname{Im} L_{2}, \operatorname{Im} L_{3}\right) \\
& =\left\{(x, y, z) \in \bar{Y} \mid \int_{0}^{1}(1-s)^{\alpha-2} x(s) \mathrm{d} s\right. \\
& \left.=\int_{0}^{1}(1-s)^{\beta-2} y(s) \mathrm{d} s=\int_{0}^{1}(1-s)^{\gamma-2} z(s) \mathrm{d} s=0\right\} .
\end{aligned}
$$

Proof. By Lemma 2, $L_{1} u=D_{0^{+}}^{\alpha} u(t)=0$ has the solution

$$
u(t)=u(0)+u^{\prime}(0) t .
$$

From the boundary conditions, we have

$$
\text { Ker } L_{1}=\{u \in X \mid u=u(0)\} \text {. }
$$

For $x \in \operatorname{Im} L_{1}$, there exists $u \in \operatorname{dom} L_{1}$ such that $x=L_{1} u \in Y$. By using the Lemma 2, we get

$$
u(t)=\frac{1}{\Gamma(\alpha)} \int_{0}^{t}(t-s)^{\alpha-1} x(s) \mathrm{d} s+u(0)+u^{\prime}(0) t .
$$

Then, we have

$$
u^{\prime}(t)=\frac{1}{\Gamma(\alpha-1)} \int_{0}^{t}(t-s)^{\alpha-2} x(s) \mathrm{d} s+u^{\prime}(0) .
$$

By the boundary value conditions of (1), we can get that $\mathrm{x}$ satisfies

$$
\int_{0}^{1}(1-s)^{\alpha-2} x(s) \mathrm{d} s=0 .
$$

On the other hand, suppose $x \in Y$ and satisfies $\int_{0}^{1}(1-s)^{\alpha-2} x(s) \mathrm{d} s=0$. Let $u(t)=I_{0^{+}}^{\alpha} x(t)$, then $u \in \operatorname{dom} L_{1}$ and $D_{0^{+}}^{\alpha} u(t)=x(t)$. Hence, $x \in \operatorname{Im} L_{1}$. Then we get

$$
\operatorname{Im} L_{1}=\left\{x \in Y \mid \int_{0}^{1}(1-s)^{\alpha-2} x(s) \mathrm{d} s=0\right\}
$$

Similarly, we have

$$
\text { Ker } L_{2}=\{v \in X \mid v=v(0)\}, \quad \operatorname{Im} L_{2}=\left\{y \in Y \mid \int_{0}^{1}(1-s)^{\beta-2} y(s) \mathrm{d} s=0\right\} \text {, }
$$

and 
Ker $L_{3}=\{w \in X \mid w=w(0)\}, \quad \operatorname{Im} L_{3}=\left\{z \in Y \mid \int_{0}^{1}(1-s)^{\gamma-2} z(s) \mathrm{d} s=0\right\}$.

Lemma 4. Let $L$ be defined by (2). Then $L$ is a Fredholm operator of index zero, $P: \bar{X} \rightarrow \bar{X}$ and $Q: \bar{Y} \rightarrow \bar{Y}$ are the linear continuous projector operators can be defined as

$$
\begin{aligned}
P(u, v, w)= & \left(P_{1} u, P_{2} v, P_{3} w\right)=(u(0), v(0), w(0)), \\
Q(x, y, z)= & \left(Q_{1} x, Q_{2} y, Q_{3} z\right) \\
= & \left((\alpha-1) \int_{0}^{1}(1-s)^{\alpha-2} x(s) \mathrm{d} s,\right. \\
& \left.(\beta-1) \int_{0}^{1}(1-s)^{\beta-2} y(s) \mathrm{d} s,(\gamma-1) \int_{0}^{1}(1-s)^{\gamma-2} z(s) \mathrm{d} s\right) .
\end{aligned}
$$

Further more, the operator $K_{P}: \operatorname{Im} L \rightarrow \operatorname{dom} L \cap \operatorname{Ker} P$ can be written by

$$
K_{P}(x, y, z)=\left(I_{0^{+}}^{\alpha} x(t), I_{0^{+}}^{\beta} y(t), I_{0^{+}}^{\gamma} z(t)\right) .
$$

Proof. Clearly, $\operatorname{Im} P=\operatorname{Ker} L$ and $P^{2}(u, v, w)=P(u, v, w)$. It follows that $(u, v, w)=((u, v, w)-P(u, v, w))+P(u, v, w)$, we have $\bar{X}=\operatorname{Ker} P+\operatorname{Ker} L$. By using simple calculation, we get that $\operatorname{Ker} L \cap \operatorname{Ker} P=\{(0,0,0)\}$. Then we have

$$
\bar{X}=\operatorname{Ker} P \oplus \operatorname{Ker} L .
$$

For $(x, y, z) \in \bar{Y}$, we have

$$
Q^{2}(x, y, z)=Q\left(Q_{1} x, Q_{2} y, Q_{3} z\right)=\left(Q_{1}^{2} x, Q_{2}^{2} y, Q_{3}^{2} z\right)
$$

By the definition of $Q_{1}$, we get

$$
Q_{1}^{2} x=Q_{1} x \cdot(\alpha-1) \int_{0}^{1}(1-s)^{\alpha-2} x(s) \mathrm{d} s=Q_{1} x .
$$

Similarly, we can show that $Q_{2}^{2} y=Q_{2} y$ and $Q_{3}^{2} z=Q_{3} z$. Thus, we can get $Q^{2}(x, y, z)=Q(x, y, z)$.

Let

$$
(x, y, z)=((x, y, z)-Q(x, y, z))+Q(x, y, z),
$$

where $(x, y, z)-Q(x, y, z) \in \operatorname{Ker} Q, \quad Q(x, y, z) \in \operatorname{Im} Q$. It follows that Ker $Q=\operatorname{Im} L$ and $Q^{2}(x, y, z)=Q(x, y, z)$, we get $\operatorname{Im} Q \cap \operatorname{Im} L=\{(0,0,0)\}$. It is clear that

$$
\bar{Y}=\operatorname{Im} L \oplus \operatorname{Im} Q .
$$

Thus

$$
\operatorname{dim} \operatorname{Ker} L=\operatorname{dim} \operatorname{Im} Q=\operatorname{codim} \operatorname{Im} L .
$$

Hence $\mathrm{L}$ is a Fredholm operator of index zero.

From the definitions of $\mathrm{P}$ and $K_{P}$, we will prove that $K_{P}$ is the inverse of $\left.L\right|_{\operatorname{dom} L \cap \operatorname{Ker} P}$. Infact, for $(x, y, z) \in \operatorname{Im} L$, we have

$$
L K_{P}(x, y, z)=\left(D_{0^{+}}^{\alpha}\left(I_{0^{+}}^{\alpha} x\right), D_{0^{+}}^{\beta}\left(I_{0^{+}}^{\beta} y\right), D_{0^{+}}^{\gamma}\left(I_{0^{+}}^{\gamma} z\right)\right)=(x, y, z) .
$$

Moreover, for $(u, v, w) \in \operatorname{dom} L \cap \operatorname{Ker} P$, we have $u(0)=v(0)=w(0)=0$ and 


$$
\begin{aligned}
K_{P} L(u, v, w) & =\left(I_{0^{+}}^{\alpha}\left(D_{0^{+}}^{\alpha} u(t)\right), I_{0^{+}}^{\beta}\left(D_{0^{+}}^{\beta} v(t)\right), I_{0^{+}}^{\gamma}\left(D_{0^{+}}^{\gamma} w(t)\right)\right) \\
& =\left(u(t)-u(0)-u^{\prime}(0) t, v(t)-v(0)-v^{\prime}(0) t, w(t)-w(0)-w^{\prime}(0) t\right),
\end{aligned}
$$

which together with the boundary condition $u^{\prime}(0)=v^{\prime}(0)=w^{\prime}(0)=0$ yields that

$$
K_{P} L(u, v, w)=(u, v, w) .
$$

From (5) and (6), we get $K_{P}=\left(\left.L\right|_{\operatorname{dom} L \cap K e r P}\right)^{-1}$.

Lemma 5. Assume $\Omega \subset \bar{X}$ is an open bounded subset such that $\operatorname{dom} L \cap \bar{\Omega} \neq \phi$, then $N$ is L-compact on $\bar{\Omega}$.

Proof. By the continuity of $f_{1}, f_{2}$ and $f_{3}$, we can get $Q N(\bar{\Omega})$ and $K_{P}(I-Q) N(\bar{\Omega})$ are bounded. By the Arzela-Ascoli theorem, we will prove that $K_{P}(I-Q) N(\bar{\Omega}) \subset \bar{X}$ is equicontinuous.

From the continuity of $f_{1}, f_{2}$ and $f_{3}$, there exist constants $M_{i}>0,(i=1,2,3)$ such that for all $(u, v, w) \in \bar{\Omega}$.

$$
\left|\left(I-Q_{1}\right) N_{1} v\right| \leq M_{1},\left|\left(I-Q_{2}\right) N_{2} w\right| \leq M_{2},\left|\left(I-Q_{3}\right) N_{3} u\right| \leq M_{3} .
$$

Furthermore, for $0 \leq t_{1}<t_{2} \leq 1,(u, v, w) \in \bar{\Omega}$, we have

$$
\begin{aligned}
\mid & \left(K_{P}(I-Q) N\left(u\left(t_{2}\right), v\left(t_{2}\right), w\left(t_{2}\right)\right)\right)-\left(K_{P}(I-Q) N\left(u\left(t_{1}\right), v\left(t_{1}\right), w\left(t_{1}\right)\right)\right) \mid \\
= & \left|K_{P}(I-Q)\left(N_{1} v\left(t_{2}\right), N_{2} w\left(t_{2}\right), N_{3} u\left(t_{2}\right)\right)-K_{P}(I-Q)\left(N_{1} v\left(t_{1}\right), N_{2} w\left(t_{1}\right), N_{3} u\left(t_{1}\right)\right)\right| \\
= & \mid\left(I_{0^{+}}^{\alpha}\left(I-Q_{1}\right) N_{1} v\left(t_{2}\right), I_{0^{+}}^{\beta}\left(I-Q_{2}\right) N_{2} w\left(t_{2}\right), I_{0^{+}}^{\gamma}\left(I-Q_{3}\right) N_{3} u\left(t_{2}\right)\right) \\
& -\left(I_{0^{+}}^{\alpha}\left(I-Q_{1}\right) N_{1} v\left(t_{1}\right), I_{0^{+}}^{\beta}\left(I-Q_{2}\right) N_{2} w\left(t_{1}\right), I_{0^{+}}^{\gamma}\left(I-Q_{3}\right) N_{3} u\left(t_{1}\right)\right) \mid \\
= & \mid I_{0^{+}}^{\alpha}\left(I-Q_{1}\right) N_{1} v\left(t_{2}\right)-I_{0^{+}}^{\alpha}\left(I-Q_{1}\right) N_{1} v\left(t_{1}\right), \\
& I_{0^{+}}^{\beta}\left(I-Q_{2}\right) N_{2} w\left(t_{2}\right)-I_{0^{+}}^{\beta}\left(I-Q_{2}\right) N_{2} w\left(t_{1}\right), \\
& I_{0^{+}}^{\gamma}\left(I-Q_{3}\right) N_{3} u\left(t_{2}\right)-I_{0^{+}}^{\gamma}\left(I-Q_{3}\right) N_{3} u\left(t_{1}\right) \mid .
\end{aligned}
$$

By

$$
\begin{aligned}
& \left|I_{0^{+}}^{\alpha}\left(I-Q_{1}\right) N_{1} v\left(t_{2}\right)-I_{0^{+}}^{\alpha}\left(I-Q_{1}\right) N_{1} v\left(t_{1}\right)\right| \\
& \leq \frac{1}{\Gamma(\alpha)}\left|\int_{0}^{t_{2}}\left(t_{2}-s\right)^{\alpha-1}\left(I-Q_{1}\right) N_{1} v(s) \mathrm{d} s-\int_{0}^{t_{1}}\left(t_{1}-s\right)^{\alpha-1}\left(I-Q_{1}\right) N_{1} v(s) \mathrm{d} s\right| \\
& \leq \frac{M_{1}}{\Gamma(\alpha)}\left[\int_{0}^{t_{1}}\left(\left(t_{2}-s\right)^{\alpha-1}-\left(t_{1}-s\right)^{\alpha-1}\right) \mathrm{d} s+\int_{t_{1}}^{t_{2}}\left(t_{2}-s\right)^{\alpha-1} \mathrm{~d} s\right]=\frac{M_{1}}{\Gamma(\alpha+1)}\left(t_{2}^{\alpha}-t_{1}^{\alpha}\right)
\end{aligned}
$$

and

$$
\begin{aligned}
& \left|\left(I_{0^{+}}^{\alpha}\left(I-Q_{1}\right) N_{1} v\right)^{\prime}\left(t_{2}\right)-\left(I_{0^{+}}^{\alpha}\left(I-Q_{1}\right) N_{1} v\right)^{\prime}\left(t_{1}\right)\right| \\
& \leq \frac{\alpha-1}{\Gamma(\alpha)}\left|\int_{0}^{t_{2}}\left(t_{2}-s\right)^{\alpha-2}\left(I-Q_{1}\right) N_{1} v(s) \mathrm{d} s-\int_{0}^{t_{1}}\left(t_{1}-s\right)^{\alpha-2}\left(I-Q_{1}\right) N_{1} v(s) \mathrm{d} s\right| \\
& \leq \frac{M_{1}}{\Gamma(\alpha-1)}\left[\int_{0}^{t_{1}}\left(\left(t_{2}-s\right)^{\alpha-2}-\left(t_{1}-s\right)^{\alpha-2}\right) \mathrm{d} s+\int_{t_{1}}^{t_{2}}\left(t_{2}-s\right)^{\alpha-2} \mathrm{~d} s\right] \\
& =\frac{M_{1}}{\Gamma(\alpha)}\left(t_{2}^{\alpha-1}-t_{1}^{\alpha-1}\right) .
\end{aligned}
$$


Similarly, we can show that

$$
\begin{aligned}
& \left|I_{0^{+}}^{\beta}\left(I-Q_{2}\right) N_{2} w\left(t_{2}\right)-I_{0^{+}}^{\beta}\left(I-Q_{2}\right) N_{2} w\left(t_{1}\right)\right| \leq \frac{M_{2}}{\Gamma(\beta+1)}\left(t_{2}^{\beta}-t_{1}^{\beta}\right), \\
& \left|\left(I_{0^{+}}^{\beta}\left(I-Q_{2}\right) N_{2} w\right)^{\prime}\left(t_{2}\right)-\left(I_{0^{+}}^{\beta}\left(I-Q_{2}\right) N_{2} w\right)^{\prime}\left(t_{1}\right)\right| \leq \frac{M_{2}}{\Gamma(\beta+1)}\left(t_{2}^{\beta-1}-t_{1}^{\beta-1}\right), \\
& \left|I_{0^{+}}^{\gamma}\left(I-Q_{3}\right) N_{3} u\left(t_{2}\right)-I_{0^{+}}^{\gamma}\left(I-Q_{3}\right) N_{3} u\left(t_{1}\right)\right| \leq \frac{M_{3}}{\Gamma(\gamma+1)}\left(t_{2}^{\gamma}-t_{1}^{\gamma}\right), \\
& \left|\left(I_{0^{+}}^{\gamma}\left(I-Q_{3}\right) N_{3} u\right)^{\prime}\left(t_{2}\right)-\left(I_{0^{+}}^{\gamma}\left(I-Q_{3}\right) N_{3} u\right)^{\prime}\left(t_{1}\right)\right| \leq \frac{M_{3}}{\Gamma(\gamma+1)}\left(t_{2}^{\gamma-1}-t_{1}^{\gamma-1}\right) .
\end{aligned}
$$

Since $t^{\alpha}, t^{\alpha-1}, t^{\beta}, t^{\beta-1}, t^{\gamma}$ and $t^{\gamma-1}$ are uniformly continuous on $[0,1]$, we have $K_{P}(I-Q) N(\bar{\Omega}) \subset X$ is equicontinuous. Thus $K_{P}(I-Q) N: \bar{\Omega} \rightarrow X$ is compact.

Lemma 6. Assume that $\left(H_{1}\right),\left(H_{2}\right)$ hold, then the set

$$
\Omega_{1}=\{(u, v, w) \in \operatorname{dom} L \backslash \operatorname{Ker} L \mid L(u, v, w)=\lambda N(u, v, w), \lambda \in(0,1)\}
$$

is bounded.

Proof. Let $(u, v, w) \in \Omega_{1}$, then $N(u, v, w) \in \operatorname{Im} L$. By (4), we get

$$
\begin{aligned}
& \int_{0}^{1}(1-s)^{\alpha-2} f_{1}\left(s, v(s), v^{\prime}(s)\right) \mathrm{d} s=0, \\
& \int_{0}^{1}(1-s)^{\beta-2} f_{2}\left(s, w(s), w^{\prime}(s)\right) \mathrm{d} s=0
\end{aligned}
$$

and

$$
\int_{0}^{1}(1-s)^{\gamma-2} f_{3}\left(s, u(s), u^{\prime}(s)\right) \mathrm{d} s=0 .
$$

Then, by integral mean value theorem, there exist constants $\xi, \eta, \zeta \in(0,1)$ such that $f_{1}\left(\xi, v(\xi), v^{\prime}(\xi)\right)=0, f_{2}\left(\eta, w\left((\eta), w^{\prime}(\eta)\right)\right)=0$ and $f_{3}\left(\zeta, u(\zeta), u^{\prime}(\zeta)\right)=0$. Then we get

$$
\begin{aligned}
& v(\xi) f_{1}\left(\xi, v(\xi), v^{\prime}(\xi)\right)=0, w(\eta) f_{2}\left(\eta, w(\eta), w^{\prime}(\eta)\right)=0 \\
& \text { and } u(\zeta) f_{3}\left(\zeta, u(\zeta), u^{\prime}(\zeta)\right)=0 .
\end{aligned}
$$

From $\left(\mathrm{H}_{2}\right)$, we get $|v(\xi)| \leq M,|w(\eta)| \leq M$ and $|u(\zeta)| \leq M$. Hence we have

$$
|u(t)|=\left|u(\zeta)+\int_{\zeta}^{t} u^{\prime}(s) \mathrm{d} s\right| \leq M+\left\|u^{\prime}\right\|_{\infty} .
$$

We obtain

$$
\|u\|_{\infty} \leq M+\left\|u^{\prime}\right\|_{\infty} .
$$

Similarly, we can show that

$$
\|v\|_{\infty} \leq M+\left\|v^{\prime}\right\|_{\infty}
$$

and

$$
\|w\|_{\infty} \leq M+\left\|w^{\prime}\right\|_{\infty} .
$$

By $L(u, v, w)=\lambda N(u, v, w)$, we get 


$$
\begin{aligned}
& u(t)=\frac{\lambda}{\Gamma(\alpha)} \int_{0}^{t}(t-s)^{\alpha-1} f_{1}\left(s, v(s), v^{\prime}(s)\right) \mathrm{d} s+u(0), \\
& v(t)=\frac{\lambda}{\Gamma(\beta)} \int_{0}^{t}(t-s)^{\beta-1} f_{2}\left(s, w(s), w^{\prime}(s)\right) \mathrm{d} s+v(0)
\end{aligned}
$$

and

$$
w(t)=\frac{\lambda}{\Gamma(\gamma)} \int_{0}^{t}(t-s)^{\gamma-1} f_{3}\left(s, u(s), u^{\prime}(s)\right) \mathrm{d} s+w(0) .
$$

Then

$$
\begin{aligned}
& u^{\prime}(t)=\frac{\lambda}{\Gamma(\alpha-1)} \int_{0}^{t}(t-s)^{\alpha-2} f_{1}\left(s, v(s), v^{\prime}(s)\right) \mathrm{d} s, \\
& v^{\prime}(t)=\frac{\lambda}{\Gamma(\beta-1)} \int_{0}^{t}(t-s)^{\beta-2} f_{2}\left(s, w(s), w^{\prime}(s)\right) \mathrm{d} s
\end{aligned}
$$

and

$$
w^{\prime}(t)=\frac{\lambda}{\Gamma(\gamma-1)} \int_{0}^{t}(t-s)^{\gamma-2} f_{3}\left(s, u(s), u^{\prime}(s)\right) \mathrm{d} s .
$$

So,

$$
\begin{aligned}
\left\|u^{\prime}\right\|_{\infty} & =\frac{1}{\Gamma(\alpha-1)} \int_{0}^{t}(t-s)^{\alpha-2}\left|f_{1}\left(s, v(s), v^{\prime}(s)\right)\right| \mathrm{d} s \\
& \leq \frac{1}{\Gamma(\alpha-1)} \int_{0}^{t}(t-s)^{\alpha-2}\left[a_{1}(s)+b_{1}(s)|v(s)|+c_{1}(s)\left|v^{\prime}(s)\right|\right] \mathrm{d} s \\
& \leq \frac{1}{\Gamma(\alpha-1)}\left[A_{1}+B_{1} M+\left(B_{1}+C_{1}\right)\left\|v^{\prime}\right\|_{\infty}\right] \int_{0}^{t}(t-s)^{\alpha-2} \mathrm{~d} s \\
& \leq \frac{1}{\Gamma(\alpha)}\left[A_{1}+B_{1} M+\left(B_{1}+C_{1}\right)\left\|v^{\prime}\right\|_{\infty}\right] .
\end{aligned}
$$

Similarly, we have

$$
\left\|v^{\prime}\right\|_{\infty} \leq \frac{1}{\Gamma(\beta)}\left[A_{2}+B_{2} M+\left(B_{2}+C_{2}\right)\left\|w^{\prime}\right\|_{\infty}\right]
$$

and

$$
\left\|w^{\prime}\right\|_{\infty} \leq \frac{1}{\Gamma(\gamma)}\left[A_{3}+B_{3} M+\left(B_{3}+C_{3}\right)\left\|u^{\prime}\right\|_{\infty}\right] .
$$

Combining (13) with (12), we get

$$
\begin{aligned}
\left\|v^{\prime}\right\|_{\infty} \leq & \frac{1}{\Gamma(\beta) \Gamma(\gamma)}\left[\left(A_{2}+B_{2} M\right) \Gamma(\gamma)+\left(B_{2}+C_{2}\right)\left(A_{3}+B_{3} M\right)\right. \\
& \left.+\left(B_{2}+C_{2}\right)\left(B_{3}+C_{3}\right)\left\|u^{\prime}\right\|_{\infty}\right] .
\end{aligned}
$$

Combining (14) with (11), we get

$$
\begin{aligned}
\left\|u^{\prime}\right\|_{\infty} \leq & \frac{1}{\Gamma(\alpha) \Gamma(\beta) \Gamma(\gamma)}\left[\left(A_{1}+B_{1} M\right) \Gamma(\beta) \Gamma(\gamma)+\left(B_{1}+C_{1}\right)\left(A_{2}+B_{2} M\right) \Gamma(\gamma)\right. \\
& \left.+\left(B_{1}+C_{1}\right)\left(B_{2}+C_{2}\right)\left(A_{3}+B_{3} M\right)+\left(B_{1}+C_{1}\right)\left(B_{2}+C_{2}\right)\left(B_{3}+C_{3}\right)\left\|u^{\prime}\right\|_{\infty}\right] .
\end{aligned}
$$


Thus, from $\frac{\Gamma(\alpha) \Gamma(\beta) \Gamma(\gamma)-\left(B_{1}+C_{1}\right)\left(B_{2}+C_{2}\right)\left(B_{3}+C_{3}\right)}{\Gamma(\alpha) \Gamma(\beta) \Gamma(\gamma)}>0$ and (14), we get

$$
\begin{aligned}
\left\|u^{\prime}\right\|_{\infty} \leq & \frac{1}{\Gamma(\alpha) \Gamma(\beta) \Gamma(\gamma)-\left(B_{1}+C_{1}\right)\left(B_{2}+C_{2}\right)\left(B_{3}+C_{3}\right)}\left[\left(A_{1}+B_{1} M\right) \Gamma(\beta) \Gamma(\gamma)\right. \\
& \left.+\left(B_{1}+C_{1}\right)\left(A_{2}+B_{2} M\right) \Gamma(\gamma)+\left(B_{1}+C_{1}\right)\left(B_{2}+C_{2}\right)\left(A_{3}+B_{3} M\right)\right] \\
:= & K_{1} \\
\left\|v^{\prime}\right\|_{\infty} \leq & \frac{1}{\Gamma(\beta) \Gamma(\gamma)}\left[\left(A_{2}+B_{2} M\right) \Gamma(\gamma)+\left(B_{2}+C_{2}\right)\left(A_{3}+B_{3} M\right)\right. \\
& \left.+\left(B_{2}+C_{2}\right)\left(B_{3}+C_{3}\right) K_{1}\right]:=K_{2}
\end{aligned}
$$

and

$$
\left\|w^{\prime}\right\|_{\infty} \leq \frac{1}{\Gamma(\gamma)}\left[A_{3}+B_{3} M+\left(B_{3}+C_{3}\right) K_{1}\right]:=K_{3} .
$$

From (8), (9) and (10), we have

$$
\|(u, v, w)\|_{\bar{X}} \leq \max \left\{K_{1}+M, K_{2}+M, K_{3}+M\right\}:=K .
$$

Hence $\Omega_{1}$ is bounded.

Lemma 7. Assume that $\left(\mathrm{H}_{3}\right)$ holds, then the set

$$
\Omega_{2}=\{(u, v, w) \mid(u, v, w) \in \operatorname{Ker} L, N(u, v, w) \in \operatorname{Im} L\}
$$

is bounded.

Proof. For $(u, v, w) \in \Omega_{2}$, we have $(u, v, w)=\left(m_{1}, m_{2}, m_{3}\right), m_{1}, m_{2}, m_{3} \in R$. Then from $N(u, v, w) \in \operatorname{Im} L$,

$$
\begin{aligned}
& \int_{0}^{1}(1-s)^{\alpha-2} f_{1}\left(s, m_{2}, 0\right) \mathrm{d} s=0, \\
& \int_{0}^{1}(1-s)^{\beta-2} f_{2}\left(s, m_{3}, 0\right) \mathrm{d} s=0
\end{aligned}
$$

and

$$
\int_{0}^{1}(1-s)^{\gamma-2} f_{3}\left(s, m_{1}, 0\right) \mathrm{d} s=0 .
$$

From $\left(\mathrm{H}_{3}\right)$ imply that $\left|m_{1}\right|,\left|m_{2}\right|,\left|m_{3}\right| \leq M^{*}$. Thus, we get

$$
\|(u, v, w)\|_{\bar{X}} \leq M^{*} \text {. }
$$

Therefore $\Omega_{2}$ is bounded.

Lemma 8. Assume that the first part of $\left(\mathrm{H}_{3}\right)$ holds, then the set

$$
\Omega_{3}=\{(u, v, w) \in \operatorname{Ker} L \mid \lambda(u, v, w)+(1-\lambda) Q N(u, v, w)=(0,0,0), \lambda \in[0,1]\}
$$

is bounded.

Proof. For $(u, v, w) \in \Omega_{3}$, we have $(u, v, w)=\left(m_{1}, m_{2}, m_{3}\right), m_{1}, m_{2}, m_{3} \in R$ and

$$
\begin{aligned}
& \lambda m_{1}+(1-\lambda)(\alpha-1) \int_{0}^{1}(1-s)^{\alpha-2} f_{1}\left(s, m_{2}, 0\right) \mathrm{d} s=0, \\
& \lambda m_{2}+(1-\lambda)(\beta-1) \int_{0}^{1}(1-s)^{\beta-2} f_{2}\left(s, m_{3}, 0\right) \mathrm{d} s=0
\end{aligned}
$$


and

$$
\lambda m_{3}+(1-\lambda)(\gamma-1) \int_{0}^{1}(1-s)^{\gamma-2} f_{3}\left(s, m_{1}, 0\right) \mathrm{d} s=0 .
$$

If $\lambda=0$, then by $\left(H_{3}\right)$, we get $\left|m_{1}\right|,\left|m_{2}\right|,\left|m_{3}\right| \leq M^{*}$. If $\lambda=1$, then $m_{1}=m_{2}=m_{3}=0$. For $\lambda \in(0,1]$, we obtain $\left|m_{1}\right|,\left|m_{2}\right|,\left|m_{3}\right| \leq M^{*}$. Otherwise, if $\left|m_{1}\right|$ or $\left|m_{2}\right|$ or $\left|m_{3}\right|>M^{*}$, from $\left(H_{3}\right)$, one has

$$
\lambda m_{1}^{2}+(1-\lambda)(\alpha-1) \int_{0}^{1}(1-s)^{\alpha-2} m_{1} f_{1}\left(s, m_{2}, 0\right) \mathrm{d} s>0
$$

or

$$
\lambda m_{2}^{2}+(1-\lambda)(\beta-1) \int_{0}^{1}(1-s)^{\beta-2} m_{2} f_{2}\left(s, m_{3}, 0\right) \mathrm{d} s>0
$$

or

$$
\lambda m_{3}^{2}+(1-\lambda)(\gamma-1) \int_{0}^{1}(1-s)^{\gamma-2} m_{3} f_{3}\left(s, m_{1}, 0\right) \mathrm{d} s>0
$$

which contradict to (15) or (16) or (17). Hence, $\Omega_{3}$ is bounded.

Remark 1 Suppose the second part of $\left(\mathrm{H}_{3}\right)$ holds, then the set

$$
\Omega_{3}^{\prime}=\{(u, v, w) \in \operatorname{Ker} L \mid-\lambda(u, v, w)+(1-\lambda) Q N(u, v, w)=(0,0,0), \lambda \in[0,1]\}
$$

is bounded.

Proof of the Theorem 1: Set $\Omega=\left\{(u, v, w) \in \bar{X} \mid\|(u, v, w)\|_{\bar{X}}<\max \left\{K, M^{*}\right\}+1\right\}$.

From the Lemma 4 and Lemma 5 we can get $\mathrm{L}$ is a Fredholm operator of index zero and $\mathrm{N}$ is L-compact on $\bar{\Omega}$. By Lemma 6 and Lemma 7, we obtain

(1) $L(u, v, w) \neq \lambda N(u, v, w)$ for every $((u, v, w), \lambda) \in[(\operatorname{dom} L \backslash \operatorname{Ker} L) \cap \partial \Omega] \times(0,1)$;

(2) $N x \notin \operatorname{Im} L$ for every $(u, v, w) \in \operatorname{Ker} L \cap \partial \Omega$.

Choose

$$
H((u, v, w), \lambda)= \pm \lambda(u, v, w)+(1-\lambda) Q N(u, v, w)
$$

By Lemma 8 (or Remark 1), we get $H((u, v, w), \lambda) \neq 0$ for $(u, v, w) \in \operatorname{Ker} L \cap \partial \Omega$. Therefore

$$
\begin{aligned}
\operatorname{deg}\left(\left.Q N\right|_{\text {KerL }}, \operatorname{Ker} L \cap \Omega, 0\right) & =\operatorname{deg}(H(., 0), \operatorname{Ker} L \cap \Omega, 0) \\
& =\operatorname{deg}(H(., 1), \operatorname{Ker} L \cap \Omega, 0) \\
& =\operatorname{deg}( \pm I, \operatorname{Ker} L \cap \Omega, 0) \neq 0 .
\end{aligned}
$$

Thus, the condition (3) of Lemma 1 is satisfied. By Lemma 1, we obtain $L(u, v, w)=N(u, v, w)$ has at least one solution in dom $L \cap \bar{\Omega}$. Hence Neumann boundary value problem (1) has at least one solution. This completes the proof.

\section{Examples}

In this section, we give two examples to illustrate our main results.

Example 1. Consider the following Neumann boundary value problem of fractional differential equation of the form 


$$
\begin{aligned}
& D_{0^{+}}^{\frac{5}{4}} u(t)=\frac{1}{8}(v(t)-6)+\frac{t^{2}}{8}\left(1+v^{\prime}(t)\right)^{\frac{1}{2}}, t \in(0,1), \\
& D_{0^{+}}^{\frac{3}{2}} v(t)=\frac{1}{6}(w(t)-4)+\frac{t^{3}}{6} \cos ^{2} w^{\prime}(t), t \in(0,1), \\
& D_{0^{+}}^{\frac{7}{4}} w(t)=\frac{1}{10}(u(t)-8)+\frac{t^{3}}{10} \sin ^{2} u^{\prime}(t), t \in(0,1), \\
& u^{\prime}(0)=u^{\prime}(1)=0, v^{\prime}(0)=v^{\prime}(1)=0, w^{\prime}(0)=w^{\prime}(1)=0 .
\end{aligned}
$$

Here $\alpha=\frac{5}{4}, \beta=\frac{3}{2}, \gamma=\frac{7}{4}$. Moreover,

$$
\begin{aligned}
& f_{1}\left(t, v(t), v^{\prime}(t)\right)=\frac{1}{8}(v(t)-6)+\frac{t^{2}}{8}\left(1+v^{\prime}(t)\right)^{\frac{1}{2}}, \\
& f_{2}\left(t, w(t), w^{\prime}(t)\right)=\frac{1}{6}(w(t)-4)+\frac{t^{3}}{6} \cos ^{2} w^{\prime}(t), \\
& f_{3}\left(t, u(t), u^{\prime}(t)\right)=\frac{1}{10}(u(t)-8)+\frac{t^{3}}{10} \sin ^{2} u^{\prime}(t) .
\end{aligned}
$$

Now let us compute $a_{1}(t), b_{1}(t), c_{1}(t)$ from $f_{1}\left(t, v(t), v^{\prime}(t)\right)$.

$$
\begin{aligned}
f_{1}\left(t, v(t), v^{\prime}(t)\right) & =\frac{1}{8}(v(t)-6)+\frac{t^{2}}{8}\left(1+v^{\prime}(t)\right)^{\frac{1}{2}} \\
& =\frac{1}{8}(v(t)-6)+\frac{t^{2}}{8}\left(1+\frac{1}{2} v^{\prime}(t)+\cdots\right) \\
& \leq \frac{1}{8}(v(t)-6)+\frac{t^{2}}{8} . \\
\left|f_{1}\left(t, v(t), v^{\prime}(t)\right)\right| & \leq \frac{1}{8}|v(t)|+\frac{7}{8} .
\end{aligned}
$$

From the above inequality, we get $a_{1}(t)=\frac{7}{8}, b_{1}(t)=\frac{1}{8}, c_{1}(t)=0$. Also,

$$
\begin{aligned}
& f_{2}\left(t, w(t), w^{\prime}(t)\right)=\frac{1}{6}(w(t)-4)+\frac{t^{3}}{6} \cos ^{2} w^{\prime}(t) \\
& \left|f_{2}\left(t, w(t), w^{\prime}(t)\right)\right| \leq \frac{1}{6}|w(t)|+\frac{5}{6} .
\end{aligned}
$$

Here, $a_{2}(t)=\frac{5}{6}, b_{2}(t)=\frac{1}{6}, c_{2}(t)=0$. Finally,

$$
\begin{aligned}
& f_{3}\left(t, u(t), u^{\prime}(t)\right)=\frac{1}{10}(u(t)-8)+\frac{t^{3}}{10} \sin ^{2} u^{\prime}(t) . \\
& \left|f_{3}\left(t, u(t), u^{\prime}(t)\right)\right| \leq \frac{1}{10}|u(t)|+\frac{8}{10} .
\end{aligned}
$$

We get, $a_{3}(t)=\frac{4}{5}, b_{3}(t)=\frac{1}{10}, c_{3}(t)=0$. And we get, $B_{1}(t)=\frac{1}{8}$,

$B_{2}(t)=\frac{1}{6}, \quad B_{3}(t)=\frac{1}{10}, \quad C_{1}(t)=C_{2}(t)=C_{3}(t)=0$. Choose $\quad M=M^{*}=8$. Also, 


$$
\begin{aligned}
& \frac{\Gamma(\alpha) \Gamma(\beta) \Gamma(\gamma)-\left(B_{1}+C_{1}\right)\left(B_{2}+C_{2}\right)\left(B_{3}+C_{3}\right)}{\Gamma(\alpha) \Gamma(\beta) \Gamma(\gamma)} \\
= & \frac{\Gamma\left(\frac{5}{4}\right) \Gamma\left(\frac{3}{2}\right) \Gamma\left(\frac{7}{4}\right)-\left(B_{1} B_{2} B_{3}\right)}{\Gamma\left(\frac{5}{4}\right) \Gamma\left(\frac{3}{2}\right) \Gamma\left(\frac{7}{4}\right)} \\
\approx & 0.73605543>0,
\end{aligned}
$$

where $\Gamma\left(\frac{1}{4}\right) \approx 3.625, \Gamma\left(\frac{3}{4}\right) \approx 1.225416702$ and $\Gamma\left(\frac{1}{2}\right)=\sqrt{\pi}$. All the conditions of Theorem 1 are satisfied. Hence, boundary value problem (18) has at least one solution.

Example 2. Consider the Neumann boundary value problem of fractional differential equation of the following form

$$
\begin{aligned}
& D_{0^{+}}^{\frac{4}{3}} u(t)=\frac{1}{17}(v(t)-15)+\frac{t^{4}}{20} \log \left(1+v^{\prime}(t)\right), t \in(0,1), \\
& D_{0^{+}}^{\frac{5}{4}} v(t)=\frac{1}{9}(w(t)-7)+\frac{t^{6}}{25}\left(1+w^{\prime}(t)\right)^{\frac{1}{3}}, t \in(0,1), \\
& D_{0^{+}}^{\frac{3}{2}} w(t)=\frac{1}{13}(u(t)-11)+\frac{t^{7}}{15} \arctan u^{\prime}(t), t \in(0,1), \\
& u^{\prime}(0)=u^{\prime}(1)=0, v^{\prime}(0)=v^{\prime}(1)=0, w^{\prime}(0)=w^{\prime}(1)=0 .
\end{aligned}
$$

Here $\alpha=\frac{4}{3}, \beta=\frac{5}{4}, \gamma=\frac{3}{2}$. Moreover,

$$
\begin{aligned}
& f_{1}\left(t, v(t), v^{\prime}(t)\right)=\frac{1}{17}(v(t)-15)+\frac{t^{4}}{20} \log \left(1+v^{\prime}(t)\right), \\
& f_{2}\left(t, w(t), w^{\prime}(t)\right)=\frac{1}{9}(w(t)-7)+\frac{t^{6}}{25}\left(1+w^{\prime}(t)\right)^{\frac{1}{3}}, \\
& f_{3}\left(t, u(t), u^{\prime}(t)\right)=\frac{1}{13}(u(t)-11)+\frac{t^{7}}{15} \arctan u^{\prime}(t) .
\end{aligned}
$$

Now let us compute $a_{1}(t), b_{1}(t), c_{1}(t)$ from $f_{1}\left(t, v(t), v^{\prime}(t)\right)$.

$$
\begin{aligned}
& f_{1}\left(t, v(t), v^{\prime}(t)\right)=\frac{1}{17}(v(t)-15)+\frac{t^{4}}{20} \log \left(1+v^{\prime}(t)\right) \\
& =\frac{1}{17}(v(t)-15)+\frac{t^{4}}{20}\left(v^{\prime}(t)-\frac{\left(v^{\prime}(t)\right)^{2}}{2 !}+\cdots\right) \\
& \left|f_{1}\left(t, v(t), v^{\prime}(t)\right)\right| \leq \frac{1}{17}|v(t)|+\frac{15}{17}+\frac{1}{20}\left|v^{\prime}(t)\right|
\end{aligned}
$$

From the above inequality, we get $a_{1}(t)=\frac{15}{17}, b_{1}(t)=\frac{1}{17}, c_{1}(t)=\frac{1}{20}$. Also,

$$
\begin{aligned}
f_{2}\left(t, w(t), w^{\prime}(t)\right) & =\frac{1}{9}(w(t)-7)+\frac{t^{6}}{25}\left(1+w^{\prime}(t)\right)^{\frac{1}{3}} \\
& =\frac{1}{9}(w(t)-7)+\frac{t^{6}}{25}\left(1+\frac{1}{3} w^{\prime}(t)+\cdots\right)
\end{aligned}
$$




$$
\begin{aligned}
\left|f_{2}\left(t, w(t), w^{\prime}(t)\right)\right| & \leq \frac{1}{9}|w(t)|+\frac{7}{9}+\frac{1}{25}\left(1+\frac{1}{3}\left|w^{\prime}(t)\right|+\cdots\right) \\
& \leq \frac{1}{9}|w(t)|+\frac{184}{225}+\frac{1}{75}\left|w^{\prime}(t)\right| .
\end{aligned}
$$

Here, $a_{2}(t)=\frac{184}{225}, b_{2}(t)=\frac{1}{9}, c_{2}(t)=\frac{1}{75}$. Similarly,

$$
\begin{aligned}
f_{3}\left(t, u(t), u^{\prime}(t)\right) & =\frac{1}{13}(u(t)-11)+\frac{t^{7}}{10} \arctan u^{\prime}(t) . \\
& =\frac{1}{13}(u(t)-11)+\frac{t^{7}}{15}\left(u^{\prime}(t)-\frac{\left(u^{\prime}(t)^{3}\right)}{3}+\cdots\right) . \\
\left|f_{3}\left(t, u(t), u^{\prime}(t)\right)\right| & \leq \frac{1}{13}|u(t)|+\frac{11}{13}+\frac{1}{15} u^{\prime}(t) .
\end{aligned}
$$

Here, $\quad a_{3}(t)=\frac{11}{13}, b_{3}(t)=\frac{1}{13}, c_{3}(t)=\frac{1}{15}$. We get, $B_{1}(t)=\frac{1}{17}, \quad B_{2}(t)=\frac{1}{9}$, $B_{3}(t)=\frac{1}{13}, \quad C_{1}(t)=\frac{1}{20}, \quad C_{2}(t)=\frac{1}{75}, \quad C_{3}(t)=\frac{1}{15}$. Choose $M=M^{*}=15$.

Also,

$$
\begin{aligned}
& \frac{\Gamma(\alpha) \Gamma(\beta) \Gamma(\gamma)-\left(B_{1}+C_{1}\right)\left(B_{2}+C_{2}\right)\left(B_{3}+C_{3}\right)}{\Gamma(\alpha) \Gamma(\beta) \Gamma(\gamma)} \\
&= \frac{\Gamma\left(\frac{4}{3}\right) \Gamma\left(\frac{5}{4}\right) \Gamma\left(\frac{3}{2}\right)-\left(\left(\frac{1}{17}+\frac{1}{20}\right)\left(\frac{1}{9}+\frac{1}{75}\right)\left(\frac{1}{13}+\frac{1}{15}\right)\right)}{\Gamma\left(\frac{4}{3}\right) \Gamma\left(\frac{5}{4}\right) \Gamma\left(\frac{3}{2}\right)} \\
& \approx 0.96872>0,
\end{aligned}
$$

where $\Gamma\left(\frac{1}{4}\right) \approx 3.625, \Gamma\left(\frac{1}{3}\right) \approx 0.1924$ and $\Gamma\left(\frac{1}{2}\right)=\sqrt{\pi}$. Hence all the conditions of Theorem 1 are satisfied. Therefore, boundary value problem (19) has at least one solution.

\section{Conclusion}

We have investigated some existence results for three-dimensional fractional differential system with Neumann boundary condition. By using Mawhin's coincidence degree theory, we established that the given boundary value problem admits at least one solution. We also presented examples to illustrate the main results.

\section{Acknowledgements}

The authors would like to thank the anonymous reviewers for their valuable comments and suggestions to improve the quality of the manuscript.

\section{References}

[1] Miller, K.S. and Ross, B. (1993) An Introduction to the Fractional Calculus and Fractional Differential Equations. Wiley, New York. 
[2] Samko, S.G., Kilbas, A.A. and Marichev, O.I. (1993) Fractional Integrals and Derivatives. Gordon and Breach Science Publishers, Yverdon.

[3] Hilfer, Z. (2000) Appliations of Fractional Calculus in Physics. World Scientific, Singapore. https://doi.org/10.1142/3779

[4] Metzler, R. and Klafter, J. (2000) Boundary Value Problems for Fractional Diffusion Equations. Physics A, 278, 107-125. https://doi.org/10.1016/S0378-4371(99)00503-8

[5] Kilbas, A.A., Srivastava, H.M. and Trujillo, J.J. (2006) Theory and Applications of Fractional Differential Equations. Elsevier, Amsterdam.

[6] Lakshmikantham, V., Leela, S. and Vasundhara Devi, J. (2009) Theory of Fractional Dynamic Systems. Cambridge Academic Publishers, Cambridge.

[7] Mainardi, A. (2010) Fractional Calculus and Waves in Linear Viscoelasticity. Imperial College Press, London. https://doi.org/10.1142/p614

[8] Abbas, S., Benchora, M. and N'Guerekata, G.M. (2012) Topics in Fractional Differential Equations. Springer, New York. https://doi.org/10.1007/978-1-4614-4036-9

[9] Zhou, Y. (2014) Basic Theory of Fractional Differential Equations. World Scientific, Singapore. https://doi.org/10.1142/9069

[10] Ahmad, B. and Nieto, J.J. (2009) Existence Results for a Coupled System of Nonlinear Fractional Differential Equations with Three-Point Boundary Conditions. Computers and Mathematics with Applications, 58, 1838-1843. https://doi.org/10.1016/j.camwa.2009.07.091

[11] Liu, Y., Ahmad, B. and Agarwal, R.P. (2013) Existence of Solutions for a Coupled System of Nonlinear Fractional Differential Equations with Fractional Boundary Conditions on the Half-Line. Advances in Difference Equations, 2013, 46. https://doi.org/10.1186/1687-1847-2013-46

[12] Aphithana, A., Ntouyas, S.K. and Tariboon, J. (2015) Existence and Uniqueness of Symmetric Solutions for Fractional Differential Equations with Multi-Point Fractional Integral Conditions. Boundary Value Problems, 2015, 68. https://doi.org/10.1186/s13661-015-0329-1

[13] Wang, Y. (2016) Positive Solutions for Fractional Differential Equation Involving the Riemann-Stieltjes Integral Conditions with Two Parameters. Journal of Nonlinear Science and Applications, 9, 5733-5740.

[14] Mawhin, J. (1993) Topological Degree and Boundary Value Problems for Nonlinear Differential Equations in Topological Methods for Ordinary Differential Equations. Lecture Notes in Mathematics, 1537, 74-142. https://doi.org/10.1007/BFb0085076

[15] Kosmatov, N. (2010) A Boundary Value Problem of Fractional Order at Resonance. Electronic Journal of Differential Equations, 135, 1-10.

[16] Bai, Z. and Zhang, Y. (2010) The Existence of Solutions for a Fractional Multi-Point Boundary Value Problem. Computers and Mathematics with Applications, 60, 2364 2372. https://doi.org/10.1016/j.camwa.2010.08.030

[17] Wang, G., Liu, W., Zhu, S. and Zheng, T. (2011) Existence Results for a Coupled System of Nonlinear Fractional 2m-Point Boundary Value Problems at Resonance. Advances in Difference Equations, 44, 1-17. https://doi.org/10.1155/2011/783726

[18] Zhang, Y., Bai, Z. and Feng, T. (2011) Existence Results for a Coupled System of Nonlinear Fractional Three-Point Boundary Value Problems at Resonance. Computers and Mathematics with Applications, 61, 1032-1047. https://doi.org/10.1016/j.camwa.2010.12.053

[19] Jiang, W. (2012) Solvability for a Coupled System of Fractional Differential Equations at Resonance. Nonlinear Analysis, 13, 2285-2292.

https://doi.org/10.1016/j.nonrwa.2012.01.023 
[20] Hu, Z., Liu, W. and Rui, W. (2012) Existence of Solutions for a Coupled System of Fractional Differential Equations. Springer, Berlin, 1-15.

https://doi.org/10.1186/1687-2770-2012-98

[21] Hu, L. (2016) On the Existence of Positive Solutions for Fractional Differential Inclusions at Resonance. SpringerPlus, 5, 957.

https://doi.org/10.1186/s40064-016-2665-8

[22] Hu, Z., Liu, W. and Chen, T. (2011) Two-Point Boundary Value Problems for Fractional Differential Equations at Resonance. Bulletin of the Malaysian Mathematical Society Series, 3, 747-755.

[23] Hu, Z., Liu, W. and Chen, T. (2012) Existence of Solutions for a Coupled System of Fractional Differential Equations at Resonance. Boundary Value Problems, 2012, 98. https://doi.org/10.1186/1687-2770-2012-98

\section{Scientific Research Publishing}

Submit or recommend next manuscript to SCIRP and we will provide best service for you:

Accepting pre-submission inquiries through Email, Facebook, LinkedIn, Twitter, etc. A wide selection of journals (inclusive of 9 subjects, more than 200 journals)

Providing 24-hour high-quality service

User-friendly online submission system

Fair and swift peer-review system

Efficient typesetting and proofreading procedure

Display of the result of downloads and visits, as well as the number of cited articles

Maximum dissemination of your research work

Submit your manuscript at: http://papersubmission.scirp.org/

Or contact am@scirp.org 